\title{
VIRTUAL SCREENING AND MOLECULAR DYNAMICS SIMULATION OF COMPOUNDS FROM THE HERBAL DATABASE OF INDONESIA AGAINST HISTONE DEACETYLASE 2
}

\author{
MUHAMMAD TEGUH SETIAWAN, ARRY YANUAR* \\ Department of Biomedical Computation, Faculty of Pharmacy, Universitas Indonesia, Depok, 16424, Indonesia. Email: arry.yanuar@ui.ac.id \\ Received: 13 July 2018, Revised and Accepted: 07 August 2018 and 10 September 2018
}

ABSTRACT

Objective: This study aimed to find the herbal compounds from the database of Indonesian herbs with potential for use as histone deacetylase 2 (HDAC2) enzyme inhibitors through virtual screening using the LigandScout program.

Methods: Virtual screening was conducted using LigandScout 4.09.3, AutodockZN, and AutoDockTools.

Results: The virtual screening process resulted in 10 compounds with the highest pharmacophore fit score rating, from which five compounds with the best criteria for molecular dynamics simulations were selected: Boesenbergin B, pongachalcone I, 6,8-diprenylgenistein, marmin, and mangostin. The $\Delta \mathrm{G}$ values obtained were, respectively, $-8.28,-9.15,-7.05,-9.07$, and -7.15 . The active crystal ligand $\mathrm{N}$-(2-aminophenyl) benzamide was used as a positive control, with $\Delta \mathrm{G}$ value of -10.27 . Molecular dynamic's simulations showed that the activity of HDAC2 inhibitors was known to interact in the amino acid residues His145C, Tyr308C, Zn379C, Leu276C, Phe155C, Phe210C, Leu144C, and Met35C.

Conclusions: Based on virtual screening and the molecular dynamics simulations, marmin was considered to provide the best overall activity of analysis. Simulation analysis of molecular dynamics from hits compound showed that analysis with MMGBSA gave higher free energy binding value than MMPBSA.

Keywords: Histone deacetylase 2, Diabetes, Herbal database Indonesia, Molecular docking, Molecular dynamics simulation.

(C) 2018 The Authors. Published by Innovare Academic Sciences Pvt Ltd. This is an open access article under the CC BY license (http://creativecommons. org/licenses/by/4. 0/) DOI: http://dx.doi.org/10.22159/ijap.2018.v10s1.52

\section{INTRODUCTION}

Histone deacetylase (HDAC) enzymes may be useful molecular targets for treating obesity in terms of metabolic modification. Studies have shown that gene regulation by HDAC can be an approach to treating type 2 diabetes and as a modification of glucose and fatty acids metabolism [1]. HDACs comprise a family of 18 enzymes that control the $\mathrm{N}-\varepsilon$-lysine deacetylation of histone to reestablish the positive charge in the $\mathrm{N}$-terminus of the histone tails [2]. In mammals, Class 1 HDAC (HDAC1, HDAC2, HDAC3, HDAC8, and HDAC10) has strong activity as a catalyst and is generally a target of HDAC2 inhibitors [3].Therefore, given the high potential of HDAC enzymes, especially HDAC Class 1, for use in diabetes treatment, further research is needed to examine various therapies to treat this disease.

Diabetes mellitus is a metabolic disorder caused by insufficient insulin production or the inability of the body to effectively use the insulin produced, resulting in an increase of glucose concentration in the blood (called hyperglycemia) [4]. This is due to the fact that insulin performs as a hormone that regulates blood sugar balance and does not work properly [5]. The prevalence of diabetes globally by 2015 is estimated to reach 416 million patients and is estimated to increase to 642 million patients by 2040 [6]. A significant change in the etiology of diabetes is contributed by environmental influences or epigenetic factors [3]. An epigenetic modification occurs due to inconsistent environmental conditions and lifestyle. At present, researchers have observed changes in epigenetic markers in diabetes models that are related to changes in gene expression due to environmental stimulation [6]. Based on the potential ability of HDAC enzymes to treat diabetes by inhibiting enzyme activity in gene overexpression, as catalysts, and for metabolism modification through interaction between enzymes and potential compounds, further research is needed to develop new drug therapies that are more effective for the treatment of diabetes. Research development of new drug therapies requires a long time and high cost if performed in vitro; hence, more work is needed to support bioinformatics-based systems that can facilitate data processing with computers.

Here, we conducted a bioinformatics-based analysis to examine molecular interactions through the simulation of molecular dynamics in search of antidiabetic potential of herbal plant database compounds found in Indonesia. We aimed to find the herbal compounds from the database of Indonesian herbs with potential for use as HDAC2 enzyme inhibitors through virtual screening using the LigandScout program. Virtual screening is another method that allows researchers to identify possible lead compounds for drug development or select some compounds in silico [7]. Virtual screening of the Indonesian medicinal herb database was performed using the website www.herbaldb. farmasi.ui.ac.id, to download compounds that could potentially work as inhibitors of HDAC2 enzymes, namely those are classified as Class I HDAC, which have strong activity as catalysts. The virtual screening compounds were then simulated molecularly to determine the interaction of tethered compounds or ligands with HDAC2 enzymes.

\section{METHODS}

We performed a virtual screening of the Indonesian herbal database using LigandScout 4.09.3 software from a pharmacophore model based on the HDAC2 structure. The resultant compounds were redocked using AutodockZN and AutoDockTools to find out values for the free binding energy and inhibition constant so that the best compound could be selected for molecular dynamics simulations. The material used was the three-dimensional (3D) structure of HDAC 2, downloaded from the Research Collaboratory for Structural Bioinformatics Protein Data Bank (PDB) website (http://www.rscb.org/pdb) with the PDB ID of 3MAX. The database used was the two-dimensional structures of candidate compounds in the form of.mol ormol2 files obtained from the Indonesian Herbal Database (www.herbaldb.farmasi.ui.ac.id). Decoy structure was obtained from the directory of useful decoys-enhanced 
website (http://www.dude.docking.org). These structures were used for validation using receiver operating characteristic curve analysis.

First, the HDAC2-based structure pharmacophore model was made. The PDB HDAC2 and PDB ID 3MAX were downloaded using LigandScout. Then, the active ligand of HDAC2 was located which was $\mathrm{N}$-(2-aminophenyl)benzamide. Ligand crystal (LLX) acted as a positive control. The 3D structures of compounds obtained from the virtual screening were then downloaded for use in the molecular docking process to acquire the free bond energy data and its inhibition constants using AutoDockTools and Autodock4Zn. Based on their free binding energy, compounds were then selected which were considered to provide the best molecular dynamics simulation.

\section{RESULTS AND DISCUSSION}

From the pharmacophore-based virtual screening, 18 compound hits were gained according to the pharmacophore fit score from LigandScout. Then, the 10 highest scoring compounds were selected to proceed with the molecular docking process, or redocking, to determine the interaction of free binding energy of the compounds to the HDAC2 macromolecule (Table 1).

Five of 10 compounds were considered to produce the best molecular dynamics simulations: Boesenbergin B, pongachalcone I, 6,8-diprenylgenistein, marmin, and mangostin. Of the five compounds, molecular dynamics analysis was performed using the Amber program, with $\mathrm{N}$-(2-aminophenyl) benzamide (LLX) ligand crystals as positive control.

Analysis of molecular dynamics: Root mean square deviation (RMSD) analysis

RMSD is a measurement to compare changes or shifts in molecular conformation. In a simulation lasting for $20 \mathrm{~ns}$, each system experienced an increase in RMSD backbone as shown in Fig. 1, which indicates that the structure of the macromolecule enzymes started to open (unfold).
Fluctuations in RMSD values could also be caused by different ligand structure forms. Ligands with large structures and large amounts of torque require more effort to achieve a stable conformation, which is shown with a stable graph as in the positive control charts of LLX ligand crystal and marmin compounds. During a simulation run, an increase in RMSD value indicates that the enzyme macromolecule structure began to unfold and the ligand would look for the corresponding binding site or appropriate coordinate of the protein. Thus, given the longer simulation time, the ability to do a more in-depth analysis of the bond stability between ligand and macromolecules was possible because achieving a stable conformation on each ligand required different durations.

\section{Analysis of molecular dynamics: Root mean square fluctuation} (RMSF) analysis

RMSF is a measure of the deviation between the particle position and some reference position. RMSF is calculated against each residue during the simulation. The RMSF scores outline the shifting conformations of each amino acid residue that gives proteins flexibility.

In the RMSF graph (Fig. 2), the back bond atom against the macromolecule HDAC2 had high fluctuations and flexibility in the residual numbers 19-28, 190-226, 262-271, and 343-361, indicating that the residue did not provide good bonding energy to the ligands of macromolecule compounds or that it did not bind at all. The instability of the interaction makes the affinity bond more unstable resulting in the value of free energy being greater.

\section{Analysis using MMPBSA}

MMPBSA is a program that can be used to calculate the free energy of a chemical bond in a molecular dynamics simulation using the PoissonBoltzmann equation [8].

MMGBSA and MMPBSA analyses were performed on the last 50 frames of 2000 simulated molecular dynamics frames. The selection of the

Table 1: Values of candidate compound's free binding energy and inhibition constants based on the Indonesian herbal database

\begin{tabular}{llllll}
\hline \multirow{2}{*}{ No. } & Compound names & Autodock4Zn & & Autodock4 & \\
\cline { 3 - 6 } & & $\begin{array}{l}\text { Free energy } \\
\text { binding (kcal/mol) }\end{array}$ & $\begin{array}{l}\text { Inhibition } \\
\text { constants/Ic (nM) }\end{array}$ & $\begin{array}{l}\text { Free energy } \\
\text { binding (kcal/mol) }\end{array}$ & $\begin{array}{l}\text { Inhibition } \\
\text { constants/Ic (nM) }\end{array}$ \\
\hline 1 & Boesenbergin B & -8.28 & 850.19 & -9.87 & 58.51 \\
2 & Pongachalcone I & -9.15 & 197.52 & -10.71 & 14.46 \\
3 & Orientanol E & -6.80 & 10.34 & -7.23 & 5.01 \\
4 & 6,8-Diprenylgenistein & -7.05 & -6.77 & -7.86 & 4.73 \\
5 & (-)-Bidwillon A & -7.00 & 7.42 & -7.24 & 4.90 \\
6 & Typhasterol & +2.38 & -7.25 & 25.51 \\
7 & Marmin & -9.07 & 222.92 & -10.38 & 1.18 \\
8 & Ovalichalcone & -6.82 & 10.05 & -8.09 & 12.14 \\
9 & Beta-mangostin & -7.38 & 3.92 & -6.71 & 432.60 \\
10 & Mangostin & -7.15 & 5.76 & -8.68 & \\
\hline
\end{tabular}

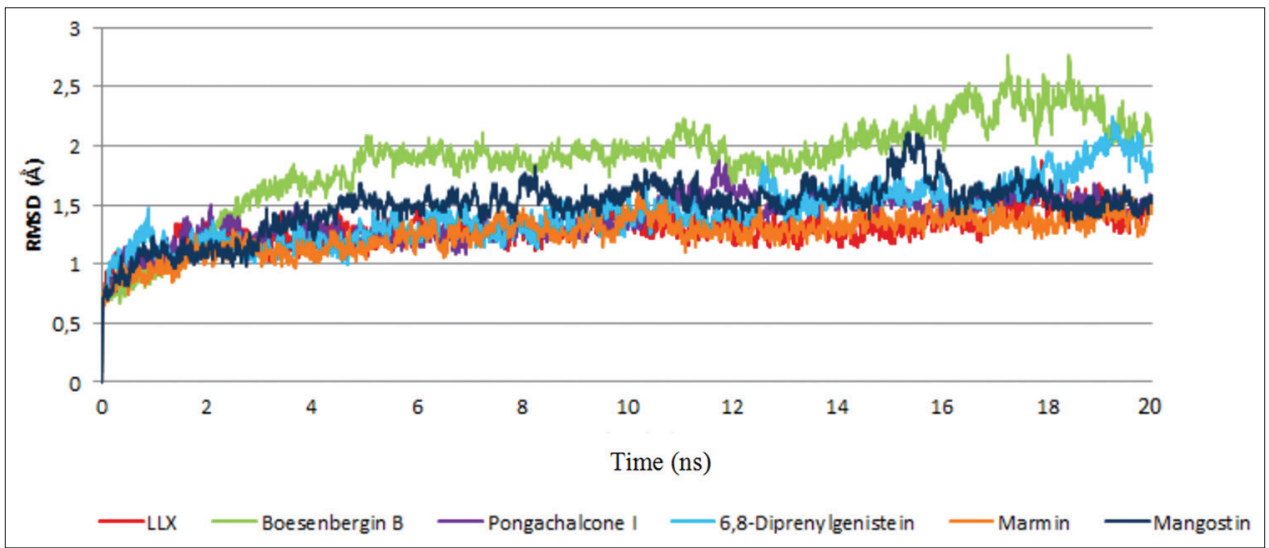

Fig. 1: Root mean square deviation versus simulation time on ligand compound simulation with histone deacetylase 2 
last 50 frames was due to the fact that in the range of these frames, the ligand bonding conditions with the compounds were considered to be stable. Boesenbergin B, pongachalcone I, and marmin had higher values of free bonding energy than the positive control of $\mathrm{N}$ - (2-aminophenyl) benzamide ligand so that they were considered to have stable binding with macromolecules. The compounds 6,8-diprenylgenistein, and mangostin, which had a free energy value lower than the positive control, did not interact stably, as shown by the fact that they took additional simulation time to achieve a stable state of interaction (Table 2).

Visualization of molecular docking results on histone deacetylase macromolecule 2 (HDAC2)

Visual analysis was performed to see the interaction between ligand and amino acid residues on macromolecule HDAC2. Visualization was done using LigandScout 4.09 .3 by inserting the ligand into the binding site on the macromolecule and then docking using the integrated AutoDock program. Visualization of the ligands interaction with macromolecules using this program was displayed in two-dimensional and 3D ways and could display good quality visual images for the benefit of publication [9]. From the interaction visualization results, the binding amino acid residues and interaction features could be compared with the LLX-positive crystal ligand control to see the interaction trend between ligand and macromolecules. Similarities of the amino acid residues and identical interaction features between ligand and positive control showed the extent of ligand interaction with macromolecules.

\section{Hydrogen bonds}

Hydrogen bonds are divided into three types based on occupancy percentages: Very weak hydrogen bonds (25-50\%), strong hydrogen bonds (50-75\%), and very strong hydrogen bonds (75-100\%) [10]. Based on those percentages, all bonds between the donor and the hydrogen bonds during simulation were recorded to determine which had $25 \%-100 \%$ occupancy since this range was considered to constitute significant bonding interactions. Based on comparisons of the six compounds (Table 3$)$, the active $\mathrm{N}$-(2-aminophenyl) benzamide ligand crystal had good hydrogen bonding interaction and good affinity values on Hie136 residues in two donors with occupancy $82.30 \%$ and $71.40 \%$; Tyr298 residue on three acceptors with occupancy $52.85 \%, 34.30 \%$, and 25.40\%; Gly144 with occupancy $47.10 \%$; and Gly295 at 39.10\%. Gly295 and Asp259 residues had occupancy of respectively $31.70 \%$ and $28.15 \%$, and interacted with boesenbergin B. Pongachalcone I interacted with the Gly133 residue on two donors with different occupancy ranges of $89.05 \%$ and $32.10 \%$.

As shown in Table 3, each compound had a number of hydrogen bonding interactions with different occupancy values. Increasing the amount of hydrogen bonds between the amino acid residues at $25-100 \%$ from the compound and the macromolecule HDAC2 will increase the hydrogen bonding interaction, especially if the interaction had a high occupancy value (the hydrogen bond was considered stable when the occupancy $>50 \%$ ) [11]. Pongachalcone I with interaction between the Lig1 donor residue and Gly133 acceptor had an occupancy value of $89.05 \%$, marmin with the Met25 donor residue and Unl1 acceptor had an occupancy value of $57.85 \%$, and mangostin on the residue Unl1 as donor and Gln21 as acceptor had an occupancy value of $54.60 \%$.

The hydrogen bond distance between the amino acid residues was analyzed using PyMol, by visualizing the macromolecule ligand structure then calculating the distance of the hydrogen bond by connecting the donor and the hydrogen bond acceptor. The results of the hydrogen bond analysis using the visual molecular dynamics program were in hbonds.dat and hbonds-details.dat format, where the hbonds.dat file showed the number of hydrogen bonds that occurred during the molecular dynamics simulation, and then, the data were plotted into graphical form using Microsoft Excel. The hbonds-details. dat file described the interaction of acceptors and hydrogen donors from amino acid residues with ligands and showed occupancy to predict the value of the hydrogen bonding interaction that occurred (Table 3). The amount of hydrogen bond interaction versus simulation time (Fig.3) show the highest number of hydrogen bond in LLX followed by Boesenbergin B. The number of hydrogen bond in LLX as the crystal

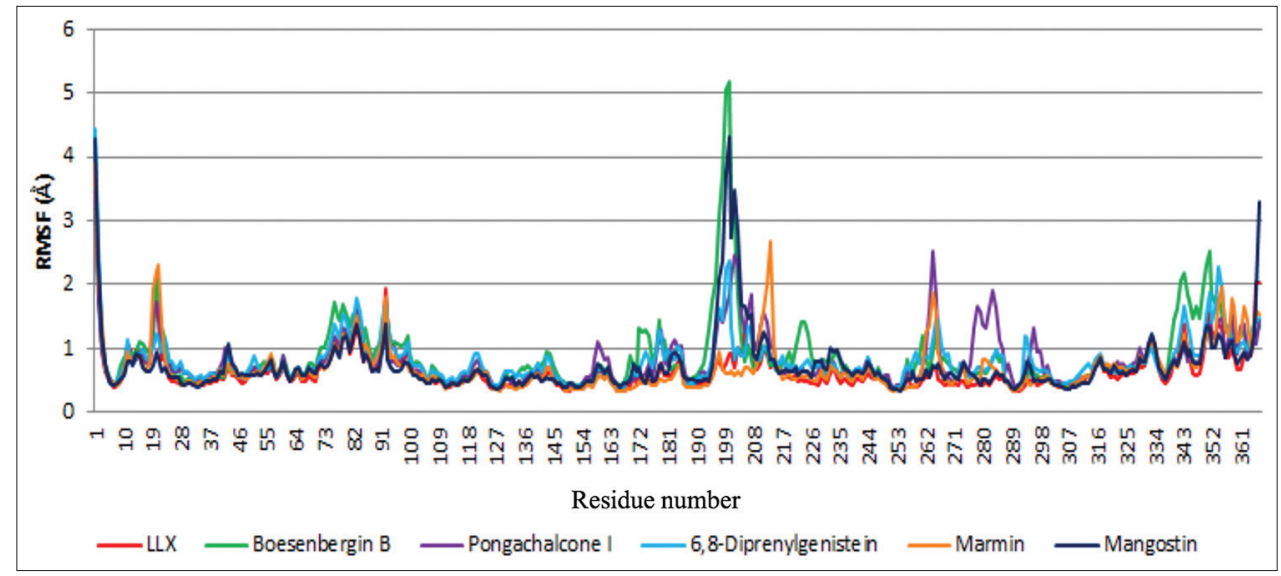

Fig. 2: Root mean square fluctuation on ligand compound simulations with histone deacetylase 2

Table 2: Values of free energy using MMGBSA, MMPBSA, and the docking of ligands and compounds against the macromolecule HDAC2

\begin{tabular}{|c|c|c|c|}
\hline Compound & $\begin{array}{l}\Delta \text { G MMGBSA } \\
\text { (kcal/mol) }\end{array}$ & $\begin{array}{l}\triangle \text { G MMPBSA } \\
\text { (kcal/mol) }\end{array}$ & $\begin{array}{l}\Delta \text { Docking G } \\
\text { (kcal/mol) Autodock4Zn }\end{array}$ \\
\hline Ligand crystal N-(2-aminophenyl) benzamide & -30.2297 & -16.9679 & -10.27 \\
\hline Boesenbergin B & -39.0787 & -25.6787 & -8.28 \\
\hline Pongachalcone I & -34.6796 & -19.5095 & -9.15 \\
\hline 6,8-Diprenylgenistein & -15.6667 & -16.7687 & -7.05 \\
\hline Marmin & -32.5167 & -23.3797 & -9.07 \\
\hline Mangostin & -20.8497 & -19.3828 & -7.15 \\
\hline
\end{tabular}

HDAC2: Histone deacetylase 2 
Table 3: Occupancy of hydrogen bond ligand complex - HDAC2

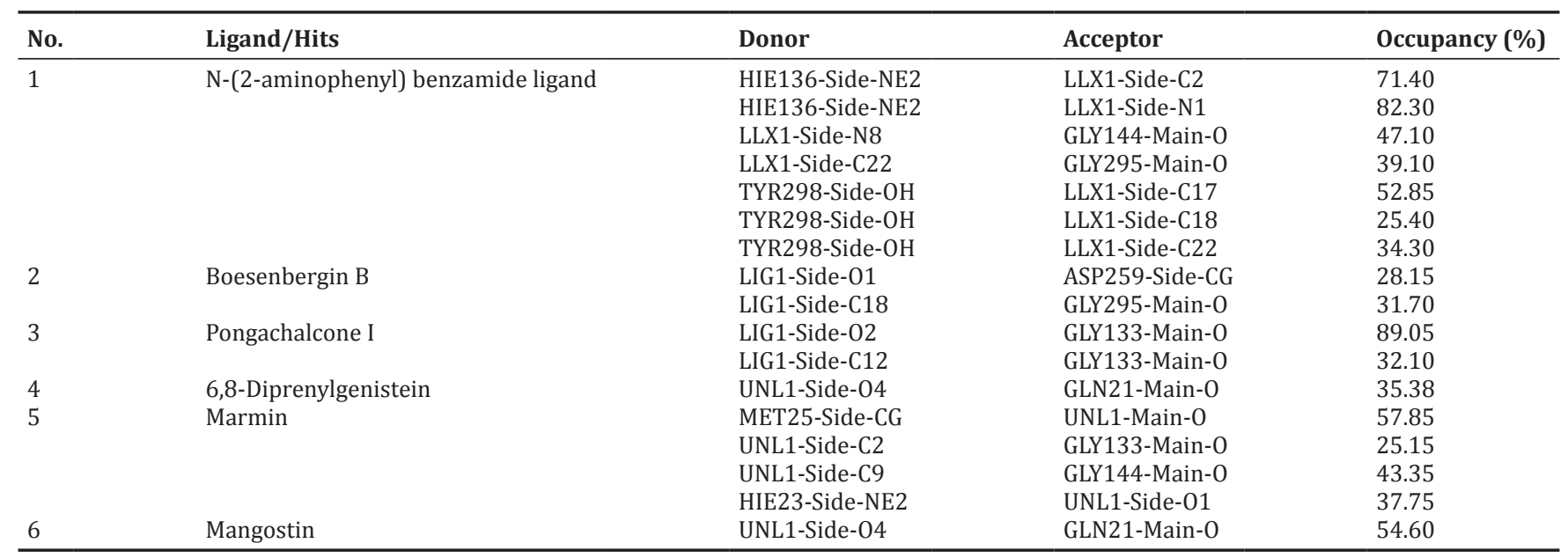

HDAC2: Histone deacetylase 2

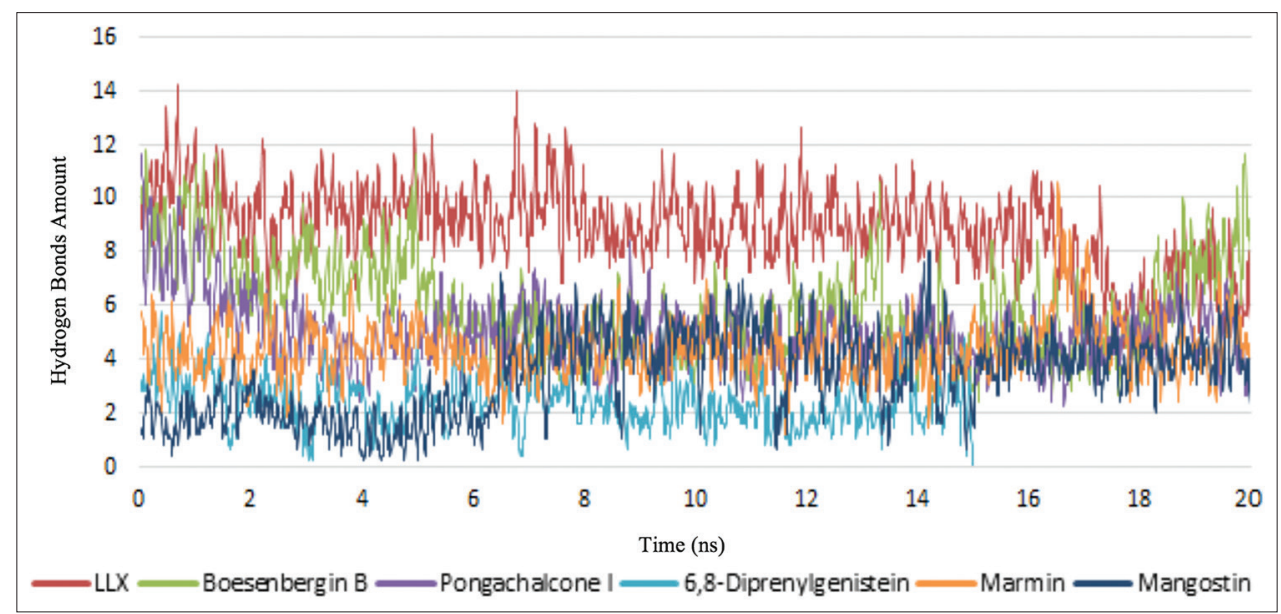

Fig. 3: Fluctuation graph of the number of hydrogen bonds versus time during simulation of molecular dynamics of ligands with macromolecules histone deacetylase 2

ligand in HDAC2 (PDB ID 3MAX) is stable during $18 \mathrm{~ns}$, but the other ligand shows fluctuation and less number of hydrogen bond.

\section{CONCLUSION}

Based on the results of virtual screening analysis of molecular docking and simulation of molecular dynamics, it could be concluded that pongachalcone I, marmin, boesenbergin $\mathrm{B}$, mangostin, and 6,8-diprenylgenistein compounds had the potential to show bond interaction with value $(\Delta G)$ were $-9.15,-9.07,-8.28,-7.15$, and -7.05 , respectively. Based on the molecular dynamics simulations of the five compounds, marmin was considered to provide the best overall activity of analysis. The marmin compounds showed similarity in amino acid residues to the positive control for Phe210C, Phe155C, Leu276C, Tyr308C, Met35C, and Leu144C and provided the best stability in the RMSD graph. HDAC2 inhibitors interacted with the essential amino acid residues and acted as the active sites of macromolecules on the residues His145C, Tyr308C, Zn379C, Leu276C, Phe155C, Phe210C, Leu144C, and Met35C. Simulation analysis of molecular dynamics from hits compound showed that analysis with MMGBSA gave higher free energy binding value than MMPBSA.

\section{ACKNOWLEDGEMENT}

All authors acknowledge Universitas Indonesia for support and PUPT Research Grants 2018.

\section{CONFLICTS OF INTEREST}

The authors declare that they have no conflicts of interest.

\section{REFERENCES}

1. Ye J. Improving insulin sensitivity with HDAC inhibitor. Diabetes 2013;62:685-7.

2. Phamte $\mathrm{H}$, Lethithu $\mathrm{H}$. Integrating structure and ligand-base approaches for modelling the histone deacetylase inhibition activity of hydroxamic acid derivatives. Asian J Pharm Clin Res 2018;11:198-206.

3. Christensen DP, Dahllöf M, Lundh M, Rasmussen DN, Nielsen MD, Billestrup N, et al. Histone deacetylase (HDAC) inhibition as a novel treatment for diabetes mellitus. Mol Med 2011;17:378-90.

4. Patil SV, Mandare AP, Pandurang GB. Study of total cholesterol (TC), tryacylglycerols (TG), high density lipoprotein cholesterol (HDL-C) in Type II diabetes mellitus. Asian J Pharm Clin Res 2017;10:116-8.

5. Ministry of Health, Republic of Indonesia. Situation and Diabetes Analysis. Jakarta: Data Center and Information, Ministry of Health, Republic of Indonesia; 2014.

6. Al-Haddad R, Karnib N, Assaad RA, Bilen Y, Emmanuel N, Ghanem A, et al. Epigenetic changes in diabetes. Neurosci Lett 2016;625:64-9.

7. TanisaAA, Riadhi R, YanuarA. Virtual screening ofbeta-secretase 1 (BASE1) inhibitors in the Indonesian herbal database as using autodock and autodock VNA. Asian J Pharm Clin Res 2017:10:148-52.

8. Fogolari F, Brigo A, Molinari H. Protocol for MM/PBSA molecular dynamics simulations of proteins. Biophys J 2003;85:159-66.

9. Wolber G, Langer T. LigandScout: 3-D pharmacophores derived from 
protein-bound ligands and their use as virtual screening filters. J Chem Inf Model 2005;45:160-9.

10. Kästner J, Loeffler HH, Roberts SK, Martin-Fernandez ML, Winn MD. Ectodomain orientation, conformational plasticity and oligomerization of erbB1 receptors investigated by molecular dynamics. J Struct Biol
2009;167:117-28.

11. Desheng L, Jian G, Yuanhua C, Wei C, Huai Z, Mingjuan J, et al. Molecular dynamics simulations and MM/GBSA methods to investigate binding mechanisms of aminomethylpyrimidine inhibitors with DPP-IV. Bioorg Med Chem Lett 2011;21:6630-5. 